\title{
Quantum metrics on noncommutative spaces
}

\author{
Maysam Maysami Sadr ${ }^{a^{*}}$ \\ ${ }^{\mathrm{a}}$ Department of Mathematics, Institute for Advanced Studies in Basic Sciences (IASBS), Zanjan, Iran \\ ${ }^{*}$ Corresponding author E-mail: sadr@iasbs.ac.ir
}

\section{Article Info}

Keywords: $C^{*}$-algebra, Enveloping von Neumann algebra, Quantum metric

2010 AMS: 46L52, 46L85, 81R60

Received: 3 March 2018

Accepted: 15 March 2018

Available online: 30 June 2018

\begin{abstract}
We introduce two new algebraic formulations for the notion of 'quantum metric on noncommutative space'. For a compact noncommutative space associated to a unital $\mathrm{C}^{*}$-algebra, our quantum metrics are elements of the spatial tensor product of the $\mathrm{C}^{*}$-algebra with itself. We consider some basic properties of these new objects, and state some connections with the Rieffel theory of compact quantum metric spaces.
\end{abstract}

\section{Introduction}

There are at least two mathematically rigorous algebraic formulations for the notion of 'quantum (noncommutative) metric space' in the literature. The famous one is due to Rieffel, and the other has been recently introduced by G. Kuperberg and N. Weaver. Following some ideas from Connes [1, Chapter VI] in noncommutative Riemannian geometry [2], Rieffel has introduced the notions of 'compact quantum metric space' and 'quantum Hausdorff-Gromov distance' [6, 7, 8]. In his theory, a compact quantum metric space $\mathfrak{q}$ is identified with the state space of a unital $\mathrm{C}^{*}$-algebra $\mathscr{A}$ (or more generally, with the state space of an order unit space) together with a weak*-compatible metric which must be induced by a 'Lipschitz seminorm' on $\mathscr{A}$ via Monge-Kantorovich's formula. Thus, in the Rieffel theory, the role of quantum metrics is played by Lipschitz seminorms. In Kuperberg-Weaver theory [4] the noncommutative space is distinguished by a von Neumann algebra $\mathscr{M} \subseteq \mathbf{L}(\mathscr{H})$ and the role of quantum metric is played by a specific one-parameter family $\left\{\mathscr{V}_{t}\right\}_{t \geq 0}$ of weak closed operator systems in $\mathbf{L}(\mathscr{H})$ such that $\mathscr{V}_{0}=\mathscr{M}^{\prime}$. This construction also can be characterized by a specific "quantum distance function" between projections of the von Neumann algebra $\mathscr{M} \bar{\otimes} \mathbf{L}\left(\ell^{2}\right)$.

The notion of 'quantum metric' recently has been considered by many authors. See $[5,9,10,11]$ and references therein. In this note, we introduce two new models for 'quantum metrics on noncommutative spaces'. Our formulations are natural translations of the concept of '(ordinary) metric' into noncommutative geometric language. In Section 2, we give our first model for quantum metrics based on 'atomic representation' of $\mathrm{C}^{*}$-algebras. We also consider some basic properties of this model. In Section 3, we show that there is no quantum metric of the first model on 'noncommutative two point space'. In Section 4, we consider a relation between our first model and the Rieffel's model of quantum metrics. In Section 5, we introduce our second model of quantum metrics.

\section{The first new model of quantum metrics}

For preliminaries on $\mathrm{C}^{*}$ and von Neumann algebras we refer the reader to [3] or [12]. Let $\mathscr{X}$ be a compact metrizable space. A function $\rho$ is a compatible metric on $\mathscr{X}$ if and only if $\rho \in \mathbf{C}\left(\mathscr{X}^{2}\right)=\mathbf{C}(\mathscr{X}) \check{\otimes} \mathbf{C}(\mathscr{X})$ and the following five conditions are satisfied for $x, y, z \in \mathscr{X}$.

(1) $\rho(x, y) \geq 0$.

(2) $\rho(x, x)=0$.

(3) If $\rho(x, y)=0$, then $x=y$.

(4) $\rho(x, y)=\rho(y, x)$.

(5) $\rho(x, y) \leq \rho(x, z)+\rho(z, y)$.

Let $\mathscr{A}$ be a unital $\mathrm{C}^{*}$-algebra. Suppose that an element $\rho \in \mathscr{A} \check{\otimes} \mathscr{A}$ deserves to be called a compatible metric on $\mathfrak{q} \mathscr{A}$. Then, $\rho$ must satisfy the following analogues of (1),(4),(5).

$\left(1^{\prime}\right) \rho \in(\mathscr{A} \check{\otimes} \mathscr{A})^{+}$. 
(4') $\mathfrak{F}(\rho)=\rho$, where $\mathfrak{F}: \mathscr{A} \check{\otimes} \mathscr{A} \rightarrow \mathscr{A} \check{\otimes} \mathscr{A}$ denotes the flip.

(5') $\mathfrak{M}(\rho) \leq \rho \otimes 1+1 \otimes \rho$, where $\mathfrak{M}: \mathscr{A} \check{\otimes} \mathscr{A} \rightarrow \mathscr{A} \check{\otimes} \mathscr{A} \check{\otimes} \mathscr{A}$ denotes the *-morphism that puts 1 in the mid position, i.e. $\mathfrak{M}(a \otimes b):=$ $a \otimes 1 \otimes b(a, b \in \mathscr{A})$.

There are many ways to state the noncommutative analogues of (2) and (3). But, it seems that the most effective and applicable way is as follows. Let $\pi: \mathscr{A} \rightarrow \mathbf{L}(\mathscr{H})$ denote a representation for $\mathscr{A}$ by bounded operators on a Hilbert space $\mathscr{H}$. We say that $\pi$ is an atomic representation if there is a family $\left\{\pi_{i}: \mathscr{A} \rightarrow \mathbf{L}\left(\mathscr{H}_{i}\right)\right\}$ of pairwise inequivalent irreducible representations of $\mathscr{A}$ such that $\pi=\oplus \pi_{i}$. (Note that our atomic representations are special cases of the atomic representations defined in [12].) Then, it follows from [3, Corollary 10.3.9] that the enveloping von Neumann algebra $\pi(\mathscr{A})^{\prime \prime}$ is equal to $\oplus_{i} \mathbf{L}\left(\mathscr{H}_{i}\right)$. Let $\pi: \mathscr{A} \rightarrow \mathbf{L}(\mathscr{H})$ be a faithful atomic representation of $\mathscr{A}$. We consider $\mathscr{A}$ as a subalgebra of $\mathbf{L}(\mathscr{H})$ and write $\mathscr{A}^{\prime \prime}$ for $\pi(\mathscr{A})^{\prime \prime}$. The characteristic function of the diagonal (w.r.t. $\pi$ ) of $\mathfrak{q} \mathscr{A} \times \mathfrak{q} \mathscr{A}$ is denoted by $P_{\delta}$ and defined to be the supremum of the family of all projections of the form $p \otimes p$ in $\mathscr{A}^{\prime \prime} \bar{\otimes} \mathscr{A}^{\prime \prime}=(\mathscr{A} \otimes \mathscr{A})^{\prime \prime} \subseteq \mathbf{L}(\mathscr{H} \bar{\otimes} \mathscr{H})$ such that $p \in \mathscr{A}^{\prime \prime}$ is a minimal projection. (In the classical case that $\mathscr{A}=\mathbf{C}(\mathscr{X})$, if we choose $\pi$ to be the reduced atomic representation then $\pi(\mathbf{C}(\mathscr{X}))^{\prime \prime}$ is isomorphic to $\ell^{\infty}(\mathscr{X})$ and $P_{\delta}$ is identified with the usual characteristic function of the diagonal of $\mathscr{X} \times \mathscr{X}$.) The analogues of (2) and (3) are as follows.

(2') $\rho P_{\delta}=P_{\delta} \rho=0$.

$\left(3^{\prime}\right)$ Let $\mathscr{H}_{\delta}$ denote the the image of the projection $P_{\delta}$ in $\mathscr{H} \bar{\otimes} \mathscr{H}$. Then, 0 is not an eigenvalue of the operator $\left.\rho\right|_{\mathscr{H}_{\delta}^{\perp}} \in \mathbf{L}\left(\mathscr{H}_{\delta}^{\perp}\right)$.

Definition 2.1. Let $\mathscr{A}$ be a unital $C^{*}$-algebra and let $\pi: \mathscr{A} \rightarrow \mathbf{L}(\mathscr{H})$ be a faithful atomic representation. A (compatible) quantum metric w.r.t. $\pi$ on $\mathfrak{q} \mathscr{A}$ is an element $\rho \in \mathscr{A} \check{\otimes} \mathscr{A}$ satisfying $\left(1^{\prime}\right)-\left(5^{\prime}\right)$. In this case, we call $(\mathscr{A}, \rho, \pi)$ a compact quantum metric space.

Let $(\mathscr{A}, \rho, \pi)$ be a compact quantum metric space. Comparing with the classical case, it is natural that we consider the value $\|\rho\|$ as the diameter of $\rho$. It is clear that if $(\mathscr{X}, \rho)$ is an ordinary compact metric space then $(\mathbf{C}(\mathscr{X}), \rho, \pi)$ is a compact quantum metric space where $\pi$ is an arbitrary atomic representation of $\mathbf{C}(\mathscr{X})$. (Indeed, it is easily checked that for any of such representation $\pi(\mathbf{C}(\mathscr{X}))^{\prime \prime}$ is isomorphic to $\ell^{\infty}\left(\mathscr{X}_{0}\right)$ where $\mathscr{X}_{0}$ is a dense subspace of $\mathscr{X}$.)

Similar to the case of ordinary metric spaces, we have the following three theorems.

Theorem 2.2. Let $\rho_{1}$ and $\rho_{2}$ be quantum metrics on $\mathfrak{q} \mathscr{A}$ w.r.t. the same representation $\pi$ of $\mathscr{A}$. Then, for every positive real number $r$, $\rho_{1}+r \rho_{2}$ is a quantum metric on $\mathfrak{q} \mathscr{A}$ w.r.t. $\pi$.

Proof. Straightforward.

Theorem 2.3. Let $\left(\mathscr{A}_{1}, \rho_{1}, \pi_{1}\right)$ and $\left(\mathscr{A}_{2}, \rho_{2}, \pi_{2}\right)$ be compact quantum metric spaces and let $r$ be a real number not less than $2^{-1} \max \left(\left\|\rho_{1}\right\|,\left\|\rho_{2}\right\|\right)$. Then, $\left(\mathscr{A}_{1} \oplus \mathscr{A}_{2}, \rho, \pi_{1} \oplus \pi_{2}\right)$ is a compact quantum metric space where $\rho=\rho_{1}+\rho_{2}+r 1_{\mathscr{A}_{1} \ddot{\otimes} \mathscr{A}_{2}}+r 1_{\mathscr{A}_{2}} \check{\otimes} \mathscr{A}_{1}$.

Proof. The conditions $\left(1^{\prime}\right)$ and $\left(4^{\prime}\right)$ are trivial for $\rho$. $\left(2^{\prime}\right)$ and $\left(3^{\prime}\right)$ follows from the fact that any minimal projection in $\left(\pi_{1} \oplus \pi_{2}\right)\left(\mathscr{A}_{1} \oplus \mathscr{A}_{2}\right)^{\prime \prime}=$ $\pi_{1}\left(\mathscr{A}_{1}\right)^{\prime \prime} \oplus \pi_{2}\left(\mathscr{A}_{2}\right)^{\prime \prime}$ is a minimal projection of $\pi_{1}\left(\mathscr{A}_{1}\right)^{\prime \prime}$ or of $\pi_{2}\left(\mathscr{A}_{2}\right)^{\prime \prime}$. Let $\mathfrak{M}, \mathfrak{M}_{1}, \mathfrak{M}_{2}$ denote the corresponding morphisms as in $\left(5^{\prime}\right)$ respectively for $\mathscr{A}_{1} \oplus \mathscr{A}_{2}, \mathscr{A}_{1}, \mathscr{A}_{2}$. With the notation $1_{i j k}:=1_{\mathscr{A}_{i} \dot{\otimes} \mathscr{A}_{j} \check{\otimes} \mathscr{A}_{k}}$ we have,

$$
\mathfrak{M}\left(\rho_{1}\right) \leq \mathfrak{M}_{1}\left(\rho_{1}\right)+2 r 1_{121}, \quad \mathfrak{M}\left(\rho_{2}\right) \leq \mathfrak{M}_{2}\left(\rho_{2}\right)+2 r 1_{212}
$$

It follows that,

$$
\begin{aligned}
\mathfrak{M}(\rho) & =\mathfrak{M}\left(\rho_{1}\right)+\mathfrak{M}\left(\rho_{2}\right)+r 1_{112}+r 1_{122}+r 1_{211}+r 1_{221} \\
& \leq \mathfrak{M}_{1}\left(\rho_{1}\right)+\mathfrak{M}_{2}\left(\rho_{2}\right)+2 r 1_{121}+2 r 1_{212}+r 1_{112}+r 1_{122}+r 1_{211}+r 1_{221} \\
& \leq \rho_{1} \otimes 1_{1}+1_{1} \otimes \rho_{1}+\rho_{2} \otimes 1_{2}+1_{2} \otimes \rho_{2}+2 r 1_{121}+2 r 1_{212}+r 1_{112}+r 1_{122}+r 1_{211}+r 1_{221} \\
& \leq \rho_{1} \otimes 1_{1}+\rho_{1} \otimes 1_{2}+\rho_{2} \otimes 1_{1}+\rho_{2} \otimes 1_{2}+r 1_{121}+r 1_{122}+r 1_{211}+r 1_{212} \\
& +1_{1} \otimes \rho_{1}+1_{2} \otimes \rho_{1}+1_{1} \otimes \rho_{2}+1_{2} \otimes \rho_{2}+r 1_{112}+r 1_{212}+r 1_{121}+r 1_{221} \\
& =\rho \otimes 1+1 \otimes \rho .
\end{aligned}
$$

Theorem 2.4. Let $\left(\mathscr{A}_{1}, \rho_{1}, \pi_{1}\right)$ and $\left(\mathscr{A}_{2}, \rho_{2}, \pi_{2}\right)$ be compact quantum metric spaces. Then, $\left(\mathscr{A}_{1} \check{\otimes} \mathscr{A}_{2}, \rho, \pi_{1} \otimes \pi_{2}\right)$ is a compact quantum metric space where

$$
\rho=\left(\rho_{1} \otimes 1_{\mathscr{A}_{2} \check{\otimes} \mathscr{A}_{2}}+1_{\mathscr{A}_{1} \check{\otimes} \mathscr{A}_{1}} \otimes \rho_{2}\right) \in\left(\mathscr{A}_{1} \check{\otimes} \mathscr{A}_{1}\right) \check{\otimes}\left(\mathscr{A}_{2} \check{\otimes} \mathscr{A}_{2}\right) \cong\left(\mathscr{A}_{1} \check{\otimes} \mathscr{A}_{2}\right) \check{\otimes}\left(\mathscr{A}_{1} \check{\otimes} \mathscr{A}_{2}\right) .
$$

Proof. Straightforward.

\section{A non-example}

In this section, we show that there is no quantum metric on the two-point noncommutative space $\mathfrak{q} \mathbf{M}_{2}$. Let $\mathscr{A}=\mathbf{M}_{2}$ be the algebra of complex $2 \times 2$ matrices. Then, $\pi=\mathrm{id}$ is an atomic representation of $\mathscr{A}$ on $\mathscr{H}=\mathbb{C}^{2}$. Let $\left\{e_{1}, e_{2}\right\}$ (resp. $\left\{f_{1}, \cdots, f_{4}\right\}$ ) denote the Euclidean basis of $\mathbb{C}^{2}$ (resp. $\mathbb{C}^{4}$ ). We identify $\mathbb{C}^{2} \otimes \mathbb{C}^{2}$ with $\mathbb{C}^{4}$ via $e_{1} \otimes e_{1} \mapsto f_{1}, e_{2} \otimes e_{2} \mapsto f_{4}, e_{1} \otimes e_{2} \mapsto f_{2}, e_{2} \otimes e_{1} \mapsto f_{3}$. Then, M $\mathbf{M}_{2} \otimes \mathbf{M}_{2}$ is identified with $\mathbf{M}_{4}$ via,

$$
\sum_{i, j, k, \ell} \lambda_{i j k \ell} 1_{i j} \otimes 1_{k \ell} \mapsto\left(\begin{array}{cccc}
\lambda_{1111} & \lambda_{1112} & \lambda_{1211} & \lambda_{1212} \\
\lambda_{1121} & \lambda_{1122} & \lambda_{1221} & \lambda_{1222} \\
\lambda_{2111} & \lambda_{2112} & \lambda_{2211} & \lambda_{2212} \\
\lambda_{2121} & \lambda_{2122} & \lambda_{2221} & \lambda_{2222}
\end{array}\right)
$$


With these identifications, $P_{\delta}$ is the projection onto the linear subspace generated by $f_{1}, f_{4}, f_{2}+f_{3}$, and hence,

$$
P_{\delta}=\left(\begin{array}{cccc}
1 & 0 & 0 & 0 \\
0 & 1 / 2 & 1 / 2 & 0 \\
0 & 1 / 2 & 1 / 2 & 0 \\
0 & 0 & 0 & 1
\end{array}\right)
$$

Suppose that $\rho \in \mathbf{M}_{4}$ satisfies ( $\left.1^{\prime}\right)-\left(4^{\prime}\right)$. Then, $\rho$ must be of the form,

$$
\rho=\left(\begin{array}{cccc}
0 & 0 & 0 & 0 \\
0 & \lambda & -\lambda & 0 \\
0 & -\lambda & \lambda & 0 \\
0 & 0 & 0 & 0
\end{array}\right)
$$

for some real number $\lambda>0$. The $8 \times 8$ matrix $M=\rho \otimes 1+1 \otimes \rho-\mathfrak{M}(\rho)$ is equal to,

$$
M=\lambda\left(\begin{array}{cccccccc}
0 & 0 & 0 & 0 & 0 & 0 & 0 & 0 \\
0 & 0 & -1 & 0 & 1 & 0 & 0 & 0 \\
0 & -1 & 2 & 0 & -1 & 0 & 0 & 0 \\
0 & 0 & 0 & 0 & 0 & -1 & 1 & 0 \\
0 & 1 & -1 & 0 & 0 & 0 & 0 & 0 \\
0 & 0 & 0 & -1 & 0 & 2 & -1 & 0 \\
0 & 0 & 0 & 1 & 0 & -1 & 0 & 0 \\
0 & 0 & 0 & 0 & 0 & 0 & 0 & 0
\end{array}\right) .
$$

For any vector $X=\left(x_{1}, \cdots, x_{8}\right) \in \mathbb{R}^{8}$ we have,

$$
\lambda^{-1}\langle M X, X\rangle=\left(x_{3}-x_{2}-x_{5}\right)^{2}+\left(x_{3}^{2}-x_{2}^{2}-x_{5}^{2}\right)+\left(x_{6}-x_{4}-x_{7}\right)^{2}+\left(x_{6}^{2}-x_{4}^{2}-x_{7}^{2}\right) .
$$

Thus $M$ is not positive and hence $\rho$ does not satisfy $\left(5^{\prime}\right)$.

Although we just mentioned a negative result on the existence of quantum metrics but it seems that there must be a huge class of quantum metrics on $\mathfrak{q} \mathbf{M}_{n}$ for $n \geq 3$.

Question 3.1. Does there exist a quantum metric on $\mathfrak{q} \mathbf{M}_{n}$ for some $n \geq 3$ ?

\section{Some relation between our first model and Rieffel's model of quantum metrics}

In this section, we consider some relations between our first model of 'compact quantum metric space' and the model introduced by Rieffel [7]. Let $(\mathscr{A}, \rho, \pi)$ be a compact quantum metric space. We are able to define a new seminorm on $\mathscr{A}$ which generalizes the Lipschitz seminorm for continuous functions on an ordinary metric space. Let $\mathscr{H}$ denote the Hilbert space of $\pi$ and let $\mathscr{H}_{\delta}$ be as in $\left(3^{\prime}\right)$. Let $\rho^{-1}$ denote the inverse of the operator $\left.\rho\right|_{\mathscr{H}_{\delta}^{\perp}} \in \mathbf{L}\left(\mathscr{H}_{\delta}^{\perp}\right)$. For any $a \in \mathscr{A}$, the Lipschitz seminorm $\|a\|_{\text {Lip }}$ are defined to be the (possibly infinite) value $\left\|(a \otimes 1-1 \otimes a) \rho^{-1}\right\|$, that is the operator norm of $(a \otimes 1-1 \otimes a) \rho^{-1}$ as an operator from the image of $\left.\rho\right|_{\mathscr{H}_{\delta}^{\perp}}$ into $\mathscr{H} \otimes \mathscr{H}$. For $a, b \in \mathscr{A}$ with $a b=b a$ the Leibnitz rule is satisfied:

$$
\begin{aligned}
\|a b\|_{\text {Lip }} & =\left\|(a b \otimes 1-1 \otimes a b) \rho^{-1}\right\| \\
& =\left\|(a b \otimes 1-a \otimes b+a \otimes b-1 \otimes a b) \rho^{-1}\right\| \\
& =\left\|[(a \otimes 1)(b \otimes 1-1 \otimes b)+(a \otimes 1-1 \otimes a)(1 \otimes b)] \rho^{-1}\right\| \\
& \leq\left\|(a \otimes 1)(b \otimes 1-1 \otimes b) \rho^{-1}\right\|+\left\|(1 \otimes b)(a \otimes 1-1 \otimes a) \rho^{-1}\right\| \\
& \leq\|a\|\|b\|_{\text {Lip }}+\|a\|_{\text {Lip }}\|b\|
\end{aligned}
$$

Also, it is clear that for any normal element $a$ we have $\|a\|_{L i p}=\left\|a^{*}\right\|_{L i p}$. The seminorm $\|\cdot\|_{L i p}$ gives rise to a semimetric on the state space $S(\mathscr{A})$ of $\mathscr{A}$ via Monge-Kantorovich formula:

$$
d(\phi, \psi):=\sup _{a^{*}=a,\|a\|_{L i p} \leq 1}|\langle\phi-\psi, a\rangle| \quad(\phi, \psi \in S(\mathscr{A})) .
$$

We give an upper bound for $d(\phi, \psi)$ in the case that $\phi$ and $\psi$ are some special pure states of $\mathscr{A}:$ Let $\pi$ be the direct sum of $\left\{\pi_{i}: \mathscr{A} \rightarrow\right.$ $\left.\mathbf{L}\left(\mathscr{H}_{i}\right)\right\}$. Suppose that $i \neq j$, and let $v$ and $w$ be two unit vectors respectively in $\mathscr{H}_{i}$ and $\mathscr{H}_{j}$. Let $\phi$ and $\psi$ be pure states on $\mathscr{A}_{\text {defined }}$ respectively by $a \mapsto\left\langle\pi_{i}(a) v, v\right\rangle$ and $a \mapsto\left\langle\pi_{j}(a) w, w\right\rangle$. Let $a$ be a self-adjoint element of $\mathscr{A}$ with $\|a\|_{L i p} \leq 1$. Since $v \otimes w \in \mathscr{H}_{\delta}^{\perp}$, we have $\|a(v) \otimes w-v \otimes a(w)\| \leq\|\rho(v \otimes w)\|$. Thus,

$$
\begin{aligned}
|\langle\phi-\psi, a\rangle|^{2} & =\langle a(v), v\rangle^{2}+\langle a(w), w\rangle^{2}-2\langle a(v), v\rangle\langle a(w), w\rangle \\
& \leq\langle a(v), a(v)\rangle+\langle a(w), a(w)\rangle-2\langle a(v), v\rangle\langle a(w), w\rangle \\
& =\langle a(v), a(v)\rangle\langle w, w\rangle+\langle a(w), a(w)\rangle\langle v, v\rangle-2\langle a(v), v\rangle\langle a(w), w\rangle \\
& =\|a(v) \otimes w-v \otimes a(w)\|^{2} \leq\|\rho(v \otimes w)\|^{2} .
\end{aligned}
$$

This shows that $d(\phi, \psi) \leq\|\rho(v \otimes w)\|$. 


\section{The second new model of quantum metrics}

As we saw above, the most problematic part of the definition of a quantum metric is the translation of conditions (2) and (3). We now translate these conditions in another way where there is no using of enveloping von Neumann algebras. Let $\mathscr{A}$ be a unital spatially continuous multiplication $\mathrm{C}^{*}$-algebra, that means the multiplication of $\mathscr{A}, m: a \otimes b \mapsto a b$, is continuous w.r.t. spatial tensor norm (e.g. $\mathscr{A}$ is abelian or finite dimensional). For $\rho \in \mathscr{A} \check{\otimes} \mathscr{A}$ satisfying (1'), consider the following conditions.

$\left(2^{\prime \prime}\right) m(\rho)=0$.

$\left(3^{\prime \prime}\right)$ For every positive element $v \in \mathscr{A} \check{\otimes} \mathscr{A}$ with $m(v)=1$ and $\mathfrak{F}(v)=v$, the element $\rho+v$ is invertible in $\mathscr{A} \check{\otimes} \mathscr{A}$.

In the case that $\mathscr{A}=\mathbf{C}(\mathscr{X})$, it is easily checked that these conditions coincide with (2),(3).

Definition 5.1. Let $\mathscr{A}$ be a unital spatially continuous multiplication $C^{*}$-algebra. An element $\rho \in \mathscr{A} \check{\otimes} \mathscr{A}$ which satisfies $\left(1^{\prime}\right),\left(2^{\prime \prime}\right)$, $\left(3^{\prime \prime}\right),\left(4^{\prime}\right),\left(5^{\prime}\right)$ is called an algebraic (compatible) quantum metric on $\mathfrak{q} \mathscr{A}$. In this case, $(\mathscr{A}, \rho)$ is called an algebraic compact quantum metric space.

Theorem 5.2. Let $\left(\mathscr{A}_{1}, \rho_{1}\right)$ and $\left(\mathscr{A}_{2}, \rho_{2}\right)$ be algebraic compact quantum metric spaces. Then, $\left(\mathscr{A}_{1} \oplus \mathscr{A}_{2}, \rho\right)$ is an algebraic compact quantum metric space where $\rho$ is as in Theorem 2.3 .

Proof. We only show that $\rho$ satisfies (3"). The other conditions are easily checked. Let $\mathscr{A}:=\mathscr{A}_{1} \oplus \mathscr{A}_{2}$. Let $m, m_{1}, m_{2}$ denote respectively the multiplications of $\mathscr{A}, \mathscr{A}_{1}, \mathscr{A}_{2}$, and let $\mathfrak{F}, \mathfrak{F}_{1}, \mathfrak{F}_{2}$ denote the corresponding flips as in $\left(4^{\prime}\right)$. We have $\mathscr{A} \check{\otimes} \mathscr{A}=\oplus_{i, j=1,2} \mathscr{A}_{i} \check{\otimes}_{\mathscr{A}}$. Let $v$ be a positive element of $\mathscr{A} \check{\otimes} \mathscr{A}$ with $m(v)=1$ and $\mathfrak{F}(v)=v$. Let $v_{i j} \in \mathscr{A}_{i} \check{\otimes}_{\mathscr{A}}$ be such that $v=\sum v_{i j}$. Then, $v_{i j}$ is positive and we have $m_{i}\left(v_{i i}\right)=1_{\mathscr{A}_{1}}$ and $\mathfrak{F}_{i}\left(v_{i i}\right)=v_{i i}$. It follows that $\rho_{i}+v_{i i}$ is invertible in $\mathscr{A}_{i} \check{\otimes}_{\mathscr{A}}$, and $r \mathscr{1}_{i} \otimes 1_{\mathscr{A}_{j}}+v_{i j}$ is invertible in $\mathscr{A}_{i} \check{\otimes} \mathscr{A}_{j}$ for $i \neq j$. Thus, $\rho+v$ is invertible in $\mathscr{A} \check{\otimes} \mathscr{A}$.

Theorem 5.3. Let $\left(\mathscr{A}_{1}, \rho_{1}\right)$ and $\left(\mathscr{A}_{2}, \rho_{2}\right)$ be algebraic compact quantum metric spaces such that $\mathscr{A}_{1}$ is commutative. Then, $\left(\mathscr{A}_{1} \check{\otimes} \mathscr{A}_{2}, \rho\right)$ is an algebraic compact quantum metric space where $\rho$ is as in Theorem 2.4.

Proof. We only show that $\rho$ satisfies $\left(3^{\prime \prime}\right)$. The other conditions are easily checked. Let $m, m_{2}$ denote respectively the multiplications of $\mathscr{A}, \mathscr{A}_{2}$, and let $\mathfrak{F}, \mathfrak{F}_{2}$ denote the corresponding flips as in (4'). Let $\mathscr{X}$ denote the Gelfand spectrum of $\mathscr{A}_{1}$. Thus, $\mathscr{A}_{1} \cong \mathbf{C}(\mathscr{X})$ and $\mathscr{A}_{1} \check{\otimes} \mathscr{A}_{2} \cong \mathbf{C}\left(\mathscr{X}, \mathscr{A}_{2}\right)$, the algebra of $\mathscr{A}_{2}$ valued continuous functions on $\mathscr{X}$. Let $v \in \mathbf{C}\left(\mathscr{X} \times \mathscr{X}, \mathscr{A}_{2} \check{\otimes} \mathscr{A}_{2}\right)$ be a positive element with $m(v)=1$ and $\mathfrak{F}(v)=v$. Then, for every $x, y \in \mathscr{X}, v(x, y)$ is positive, $m_{2}(v(x, y))=1_{\mathscr{A} 2}$, and $\mathfrak{F}_{2}(v(x, y))=v(x, y)$. Thus, $\rho_{2}+v(x, y)$ is invertible in $\mathscr{A}_{2} \check{\otimes} \mathscr{A}_{2}$. It follows that $1_{\mathscr{A}_{1} \otimes \mathscr{A}_{1}} \otimes \rho_{2}+v$ (which is equal to the function $(x, y) \mapsto \rho_{2}+v(x, y)$ ) is invertible. Thus, $\rho+v$ is also invertible.

The main gap in our work is the lack of a nonclassical example:

Problem 5.4. Give an example of a nonclassical (algebraic) quantum metric.

\section{References}

[1] A. Connes, Noncommutative geometry, Academic Press, 1994.

[2] A. Connes, On the spectral characterization of manifolds, J. Noncommutative Geometry 7 (1) (2013): 1-82.

[3] R.V. Kadison, J.R. Ringrose, Fundamentals of the theory of operator algebras: Advanced theory, Vol. 2, American Mathematical Soc., 1997.

[4] G. Kuperberg, N. Weaver, A von Neumann algebra approach to quantum metrics/quantum relations, Vol. 215, no. 1010. American Mathematical Society, 2012 .

[5] P. Martinetti, From Monge to Higgs: a survey of distance computations in noncommutative geometry, Noncommutative Geometry and Optimal Transport 676, 2016.

[6] M.A. Rieffel, Metrics on state spaces, Doc. Math. 4 (1999): 559-600.

[7] M.A. Rieffel, Group $C^{*}$-algebras as compact quantum metric spaces, Doc. Math. 7 (2002): 605-651.

[8] M.A. Rieffel, Gromov-Hausdorff distance for quantum metric spaces/Matrix algebras converge to the sphere for quantum Gromov-Hausdorff distance, Vol. 168, no. 796. American Mathematical Soc., 2004.

[9] M.M. Sadr, Metric operator fields. (arXiv:1705.03378 [math.OA])

[10] M.M. Sadr, Vietoris topology on hyperspaces associated to a noncommutative compact space, Mathematica, 60 (83) (1) (2018): $72-82$.

[11] M.M. Sadr, Quantum metric spaces of quantum maps, Universal Journal of Mathematics and Applications, 1 (1) (2018): 54-60.

[12] M. Takesaki, Theory of operator algebras I, Reprint of the first (1979) edition, Encyclopaedia of Mathematical Sciences, 124, Operator Algebras and Noncommutative Geometry, 5. (2002). 